\title{
Pyrolysis derivatization of humic substances \\ 1. Pyrolysis of fulvic acids in the presence of tetramethylammonium hydroxide
}

\author{
F. Martin *, F.J. González-Vila, J.C. del Rio and T. Verdejo \\ Instituto de Recursos Naturales y Agrobiologio. C.S.I.C., P.O. Box 1052, 41080 -Sevilla \\ (Spain)
}

\begin{abstract}
Pyrograms of three fulvic acids (FAs) of different origins and the corresponding pyrograms obtained by reactive pyrolysis with tetramcthylammonium hydroxide (TMAH) are compared. This procedure introduces both striking qualitative and quantitative changes in the pyrolytic patterns of the FAs, which are discussed in terms of the usefulness of this approach to structural studies. Some non-pyrolysis compounds (probably retained in the macromolecular fulvic matrix) seem to be released when the FAs are pyrolysed in the presence of TMAH. A set of benzenocarboxylic acid standards was also pyrolysed both in the absence and in the presence of TMAH. The results obtained suggest a thermally assisted chemolysis rather than in situ methylation of the pyrolysis products.
\end{abstract}

Futvic acids; humic substances; pyrolysis; tetramethylammonium hydroxide.

\section{INTRODUCTION}

Humic substances (HS) are widely distributed in almost all terrestrial and aquatic environments. Owing to their colloidal properties and high functionality they play important roles in reactions and processes related to the conservation and production of soil ecosystems, which have been recently reviewed $[1,2]$.

The complex, variable composition of the fulvic acids (FAs) (the alkali and acid-soluble collodial humic fraction) has been investigated by many different methods with divergent results. Overall the structural information gained by analytical pyrolysis (Py) of FA is unexpectedly much lower than that given by the other humic fractions (humic acids and humins). Thus, FA gives low yields of Py compounds, with major components being characieristic of soil polysaccharides, and a small number of aromatic compounds, whereas Py of humic acids gives much richer pyrograms. This 
behaviour of FAs seems to be related to their particularly stable macromolecular conformation and more importantly to their highly acidic character. As stated by Anderson and Winans [3], pyrolysis of underivatized carboxylic groups, such as those present in the FA structure, poses serious analytical problems.

The introduction of structural changes in humic macromolecules by chemical treatments has previously been used to gain insight into the mechanisms of the pyrolytic breakdown of HS [4-6]. The specific modification of carboxylic groups, usually based on the preparation of ester derivatives, has not always been satisfactorily accomplished. In general, the esterification conditions are either too drastic (when methanol $\mathrm{HCl}$ is used) or suffer from poor yields and non-specificity (when diazomethane is employed). This modification is responsible for changes not only in the solubility and colloidal properties, but probably also in the nature of the different intramolecular forces affecting the structural arrangement of the macromolecular consituents.

In the present paper we report the advantages of the pyrolysis of FAs in the presence of tetramethylammonium hydroxide (TMAH) to assess their macromolecular structure. This procedure, also called simultaneous pyrolysis methylation (SPM), was introduced by Challinor [7-9] and has been applied to different natural and artificial polymers such as lignins, cutins, alkyd resins and polyester fibres [3,7-9]. The process, considered to be a high-temperature hydrolytic methylation reaction, produces methyl esters of carboxylic acids and methyl ethers of hydroxy compounds. Recently, de Leeuw and Bass [10] also demonstrated with aliphatic esters that this procedure consists of a thermally assisted chemolysis (TAC).

The aims of this work, the first of a series, are to assess whether this procedure could provide a better understanding of the FA structure based upon pyrolysis techniques, and to what extent TAC instead of SPM could take place when pyrolysis is performed in the presence of TMAH.

\section{MATERIAL AND METHODS}

The FA samples were isolated from particulate matter of a water-logged peatland (FA-P), and from two Podsol soils in Vollbüttel, Germany (FA-V) and Armadale, Canada (FA-A). The isolation procedures were as follows: the FA-P was obtained by shaking the above water with a strong-acid chelating resin; after $24 \mathrm{~h}$ the solution, with a $\mathrm{pH}$ of 1.5 , was separated by filtration, centrifuged, concentrated by vacuum distillation and finally lyophilized. The Podsol FAs were extracted by $0.5 \mathrm{~N} \mathrm{NaOH}$ under $\mathrm{N}_{2}$; at pH $4 \mathrm{n}$ FA-Fe complex was precipitated which was then separated by centrifugation, dialyzed and shaken by a strong-acid chelating resin following the same treatment as above. The analytical data of the three samples have been previously reported $[11-13]$. 
The FA samples were placed in a quartz tube and pyrolysed in a CDS (Chemical Data System) Pyroprobe at $700^{\circ} \mathrm{C}$ for $10 \mathrm{~s}$. For pyrolysis in the presence of TMAH, the FA samples were first dissolved in the minimum amount of TMAH (25\% aqueous solution) and dried in a desiccator overnight. The syrups were placed on the ribbon foil of the CDS pyroprobe and heated to $700^{\circ} \mathrm{C}$ for $10 \mathrm{~s}$. Separation of the pyrolysis products was achieved by two different fused silica colümns (SE-52 and FFAP) of $25 \mathrm{~m}$ length and $0.2 \mathrm{~mm}$ i.d. The gas chromatograph (Hewlett Packard HP-5890) was programmed from $40^{\circ} \mathrm{C}$ to $270^{\circ} \mathrm{C}$ at a rate of $6^{\circ} \mathrm{C} / \mathrm{min}$. Helium was used as carrier gas. The mass spectrometer (HP 5988A) was set at $70 \mathrm{eV}$. Identification was achieved by mass fragmentography, library search and comparison with literature data.

\section{RESULTS AND DISCUSSION}

The total ion chromatograms (TICs) of the pyrolysis products obtained from the three FAs in the absence and in the presence of TMAH are compared in Figs. 1-3. The TICs of Fig. 3 for FA-A were obtained by separating the mixtures with a polar column (FFAP). The numbers in the Figures refer to the compounds listed in Tables 1 and 2.

In general there is good correspondence between most of the compounds originated in the FA pyrolysis and those obtained in the presence of

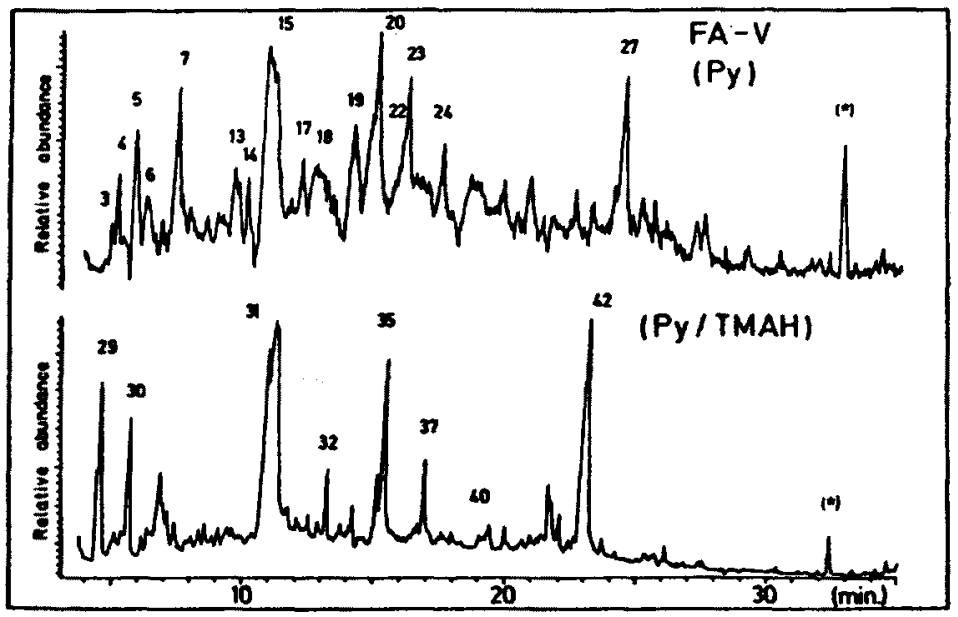

Fig. I. Total ion chromatogram of the thermal degradative products obtained after pyrolysis of FA.V (a) in the absence of TMAH, and (b) in the presence of TMAH. Numbers refer to Tables 1 and 2. (") Phthalate contaminants. 


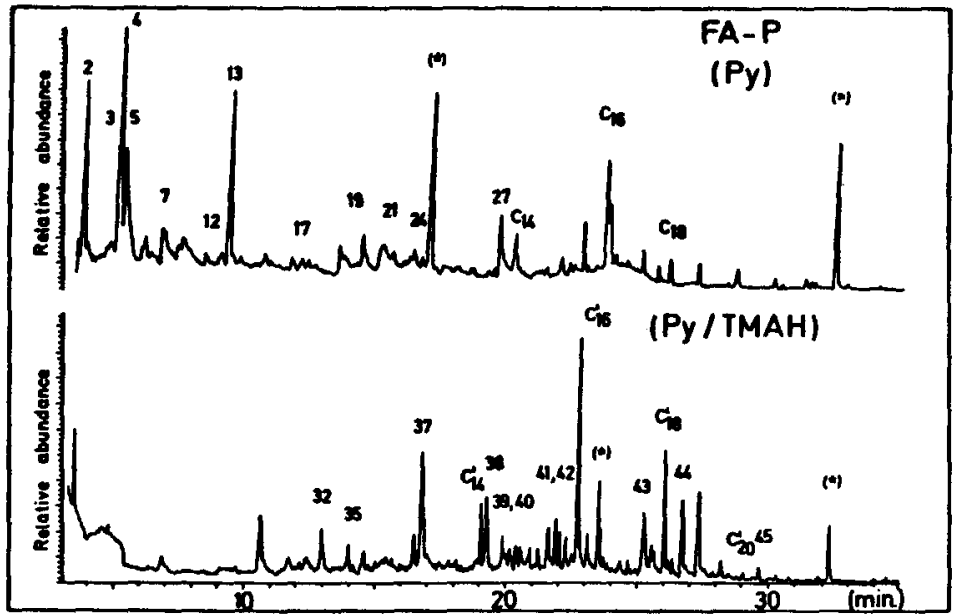

Fig. 2. Total ion chromatogram of the thermal degradative products obtained after pyrolysis of FA-P (a) in the absence of TMAH, and (b) in the presence of TMAH. Numbers refer to Tables 1 and 2. Fatty acids are denoted as $C_{14}, C_{16}, C_{18} \ldots$, and fatty acid methyl esters as $\mathrm{C}_{14}, \mathrm{C}_{16}^{\prime}, \mathrm{C}_{18} \ldots$ (*) $^{*}$ Phthalate contaminants.

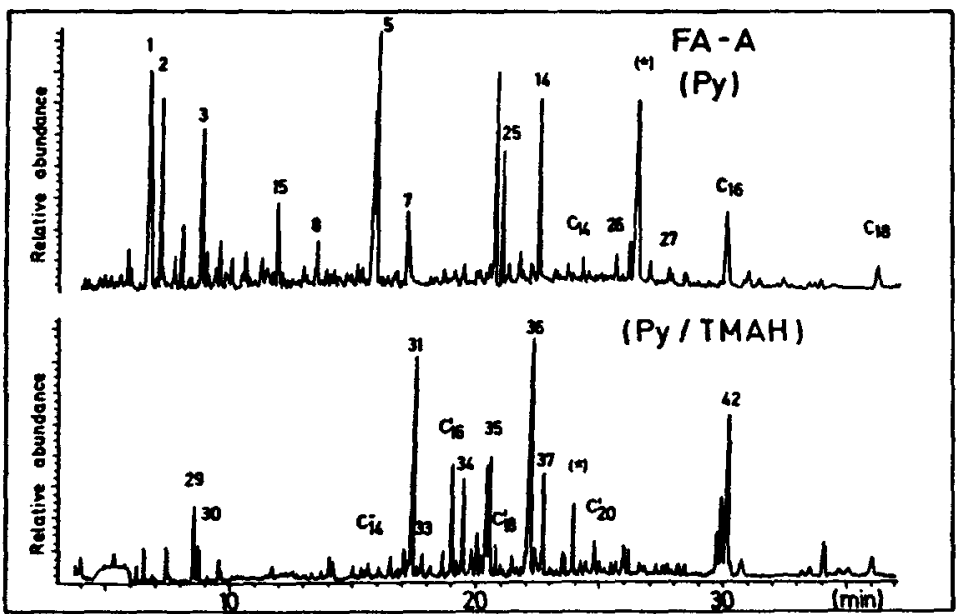

Fig. 3. Total ion chromatogram (FFAP gas chromatographic column) of the thermal degradative products obtained after pyrolysis of FA-A (a) in the absence of TMAH, and (b) in the presence of TMAH. Numbers refer to Tables $I$ and 2. Fatty acids are denoted as $C_{14}$, $\mathrm{C}_{16}, \mathrm{C}_{18} \ldots$ and fatty acid methyl esters as $\mathrm{C}_{16}^{\prime}, \mathrm{C}_{16}^{\prime}, \mathrm{C}_{18}^{\prime} \ldots\left(^{*}\right)$ Phthalate contaminants. 
TABLE I

Main thermal degradative products identfied in the pyrolysates of the untreated fulvic samples

\begin{tabular}{|c|c|c|c|c|}
\hline & Pyrolysis products & FA-V & FA-A & FA-P \\
\hline 1 & Acetic acid & + & +++ & + \\
\hline 2 & Furfural & - & +++ & - \\
\hline 3 & Methylfurfural & $-\quad+$ & $++t$ & ++ \\
\hline 4 & Dimethyltrisulphide & $+t$ & - & +++ \\
\hline 5 & Phenol & $+t+$ & $+t+$ & ++ \\
\hline 6 & 2-Furancarboxylic acid & + & - & - \\
\hline 7 & Cresols & ++ & ++ & + \\
\hline 8 & Guiacol & $+t$ & + & + \\
\hline 9 & Furanmethanol & + & ++ & - \\
\hline 10 & Benzenediol & + & - & - \\
\hline 11 & 2-Methyl-1 $H$-isoindol-1,3(2H)-dione & + & - & - \\
\hline 12 & 2-Methyl-3-hydroxy-pyran-4-one & - & - & + \\
\hline 13 & Dimethyltetrasulphide & + & - & $++t$ \\
\hline 14 & Benzoic acid & + & + & + \\
\hline 15 & 2-Butenoic acid & - & ++ & - \\
\hline 16 & Benzenediol & $+t+$ & - & - \\
\hline 17 & 1,3-Benzofurandione & ++ & - & + \\
\hline 18 & $2,2^{\prime}$-Bifuran & + & - & - \\
\hline 19 & Methyl-1,3-bensofurandione & $+t$ & - & + \\
\hline 20 & Benzopyran-2-one & $+t$ & - & - \\
\hline 21 & Methoxy-1,3-benzofurandione & - & - & + \\
\hline 22 & I $H$-Isoindol-1,3-2(H)-dione & + & - & - \\
\hline 23 & Dibenzofuran & + & - & + \\
\hline 24 & Dimethyl-1,3-benzofurandione & $+t$ & - & + \\
\hline 25 & 4-Oxopentenoic acid & - & $t+$ & - \\
\hline 26 & Acetovanillone & - & + & - \\
\hline 27 & Biphenol & - & + & - \\
\hline \multirow[t]{2}{*}{28} & Dibenzofuranol & $+t$ & - & - \\
\hline & Fatty acids $C_{14}-C_{18}$ & $+t$ & ++ & $+t$ \\
\hline
\end{tabular}

(-) not detected, $(+)$ low abundance, $(t+)$ medium abundance, $(+t+)$ high abundance

originated in the FA pyrolysis and those obtained in the presence of TMAH. Thus, the group of 1,3-iso-benzofurandione derivatives produced after conventional pyrolysis is converted into a series of substituted benzenecarboxylic acid methyl esters with two carboxyl groups in the ortho position, whereas the free fatty acids become the corresponding fatty acid methyl ester series after pyrolysis in the presence of TMAH.

It is interesting to note that many of the compounds identified after pyrolysis in the presence of TMAH are the same as those produced by oxidation with persulphate and subsequent methylation [11,14]. As far as we know, this is the first time that benzenecarboxylic acids, proposed as building blocks of the FA structure by Schnitzer and Kahn [15], have been 
Main thermal degradative products identified in the pyrolysates of the fulvic samples treated with TMAH

Phyrolysis product

FA-V FA-A FA-P

29 Phosphoric acid trimethyl ester

30 Butanedioic acid dimethyl ester

31 Permethylated monosaccharide

32 Methoxybenzenecarboxylic acid, methyl ester

33 Dichlorobenzenocarboxylic acid, methyl ester

34 Permethylated monosaccharide

35 Benzendicarboxylic acid dimethyl ester

36 Unknown

37 Dimethoxybenzenecarboxylic acid methyl ester

38 Trimethoxybenzenecarboxylic acid methyl ester

39 Dimethoxydimethyl benzenecarboxylic acid methyl ester

40 Methoxybenzenedicarboxylic acid methyl ester

41 Dimethoxybenzenedicarboxylic acid dimethyl ester

42 Benzenetricarboxylic acid trimethyl ester

43 Methoxybenzenetricarboxylic acid trimethyl ester

44 Benzenetetracarboxylic acid tetramethyl ester

45 Methoxybenzenetetracarboxylic acid tetramethyl ester

46 Benzenepentacarboxylic acid pentamethyl ester

Falty acid methyl esters $C_{10}-C_{24}$

\begin{tabular}{|c|c|c|}
\hline++ & ++ & - \\
\hline++ & + & - \\
\hline$+t$ & +++ & - \\
\hline 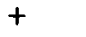 & + & ++ \\
\hline+ & + & $t+$ \\
\hline - & ++ & - \\
\hline$+t$ & $++t$ & + \\
\hline - & $++t$ & - \\
\hline+ & $+t$ & $++t$ \\
\hline- & - & $+t$ \\
\hline- & - & + \\
\hline+ & + & + \\
\hline - & - & $+t$ \\
\hline++ & $+t+$ & ++ \\
\hline - & - & $+t$ \\
\hline+ & + & $t+$ \\
\hline - & - & + \\
\hline- & - & + \\
\hline & ++ & $+t+$ \\
\hline
\end{tabular}

(-) not detected, $(+)$ low abundance, $(++)$ medium abundance, $(+++)$ high abundance.

detected by pyrolysis techniques. In order to assess the behaviour of benzenecarboxylic acids against pyrolysis, a set of experiments with benzenecarboxylic acid standards was performed. Table 3 shows the results of the pyrolysis of the benzenecarboxylic acid standards both in the absence and in the presence of TMAH. The benzenedicarboxylic and benzenetricarboxylic acids produced predominantly benzoic acid after pyrolysis. Only in the case in which two carboxylic groups are located in the ortho position was the corresponding anhydride (1,3-isobenzofurandione) formed. These experiments show the disadvantage of the pyrolysis technique for the structural assessment of macromolecules based on benzenecarboxylic acid moieties, since these are decarboxylate and not released upon pyrolysis.

However, pyrolysis in the presence of TMAH introduces a new strategy for the structural assessment of macromolecules since it releases the benzenecarboxylic acid moieties, as methyl esters, upon pyrolysis. In all cases, the products obtained after pyrolysis in the presence of TMAH of the standards were the methyl esters of the original benzenecarboxylic acids. The absence of benzoic acid methyl ester and 1,3-benzofurandione in this case reinforces the high-temperature hydrolysis and alkylation mechanism postulated by Challinor [7-9], and is in agreement with the idea stated by 
TABLE 3

Main thermal degradative products obtained after pyrolysis of standard compounds in the absence and in the presence of TMAH

Standard compound

Pyrolysis product Pyrolysis/TMAH products

1,2-Benzenedicarboxylic acid

1,3-Benzenedicarboxylic acid

1,4-Benzenedicarboxylic acid

1,2,4-Benzenetricarboxylic acid

1,3,5-Benzenetricarboxylic acid
1,3-Benzofurandione

Benzoic acid

Benzoic acid

Benzoic acid +

1,3-benzofurandione

Benzoic acid
1,2-Benzenedicarboxylic acid dimethyl ester

1,3-Benzenedicarboxylic acid dimethyl ester

4,4-Benzenedicarboxylic acid dimethyl ester

1,2,4-Benzenetricarboxylic acid trimethyl ester

1,3,5-Benzenetricarboxylic acid trimethyl ester<smiles>O=C(O)c1ccccc1C(=O)O</smiles><smiles>O=C(O)c1cccc(C(=O)O)c1</smiles>

I MAH

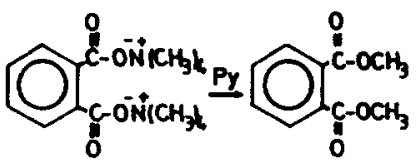

(a)<smiles>COC(OC)c1cccc([C@](C)(O)C(=O)N(C)C)c1</smiles>

(b)

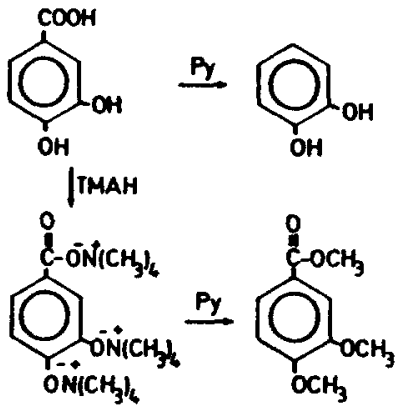

(c)

Fig. 4. Pyrolysis behaviour of the benzenecarboxylic acids in the absence and in the presence of TMAH: (a) 1,2-benzenedicarboxylic acid, (b) 1,3-benzenedicarboxylic acid. (c) Mechanism suggested for the pyrolysis of benzenediolcarboxylic acid. 
de Leeuw and Baas [10] of a TAC rather than a methylation of the pyrolysis products. The mechanisms suggested for the pyrolytic behaviour of the benzenecarboxylic acids in the absence and in the presence of TMAH are indicated in Figs. $4(\mathrm{a})$ and $4(\mathrm{~b})$.

The pyrogram of FA-V (Fig. I(a)) consists mainly of pyrolysis products of polysaccharides (such as furan derivatives), lignin pyrolysis products (such as phenolic compounds), and a series of 1,3-isobenzofurandione structures. The alteration of the pyrolysate pattern after pyrolysis in the presence of TMAH (Fig. 1(b)) is apparent in this sample. The release of large amounts of benzenecarboxylic acids (as methyl esters) and of a compound with a mass spectrum characterized by the fragments at $m / z 88$ and 101 , suggesting a permethylated monosaccharide, is noticeable.

It is noteworthy that the polysulphide compounds (dimethyltrisulphide and dimethyltetrasulphide) produced by direct pyrolysis of FA-V and FA-P are absent in the pyrolysate obtained in the presence of TMAH. This might be due to a reaction of TMAH at the pyrolysis temperature with the $\mathrm{C}-\mathrm{S}$ and S-S bonds of the originally organic sulphur moiety, preventing the formation of polysulphide chains. This would also be in agreement with a TAC.

A benzenediol was identified as a major pyrolysis product of FA-V. However, pyrolysis of the FA-V sample in the presence of TMAH did not produce dimethoxybenzene, as expected, but a dimethoxybenzenecarboxylic acid (as methyl ester). This suggests that the original moiety present in the structure of the FA-V sample is a benzenediolmonocarboxylic acid, which undergoes decarboxylation upon pyrolysis (as has been demonstrated with standards) and yields benzenediol. Pyrolysis in the presence of TMAH prevents decarboxylation and yields the methyl ester of the dimethoxybenzenecarboxylic acid. The mechanism is indicated in Fig. 4(c). The absence of dimethoxybenzene in this case is again in agreement with a TAC rather than a methylation of the pyrolysis products. This is also the case of the phenols produced upon pyrolysis, which are not converted to methoxybenzene after pyrolysis in the presence of TMAH. The original structural moieties may be monohydroxybenzenecarboxylic acids, which suffer decarboxylation and yield phenol upon pyrolysis.

The presence of some new compounds, such as phosphoric acid trimethyl ester, is somewhat interesting. This compound could arise from the breakdown of the organic phosphate present in the soils [16]. Phosphate moieties have not previously been detected by pyrolysis techniques.

Pyrolysis of the FA-P (Fig. 2(a)) sample released only a few compounds, giving little information on its structure. The major compounds detected were dimethyltrisulphide and dimethyltetrasulphide, with minor amounts of phenol, cresols, fatty acids and a series of 1,3-isobenzofurandione structures. However, pyrolysis in the presence of TMAH (Fig. 2(b)) released more compounds, benzenecarboxylic acids and fatty acids (as their methyl esters) being the most predominant ones. 
The pyrogram of the FA-A sample obtained with a polar column (Fig. 3(a)) shows similar pyrolysis compounds to FA-A. The pyrolysis of FA-A in the presence of TMAH showed similar trends to those reported in the case of FA-V, with the release of the methyl esters of benzenecarboxylic and fatty acids. Again, some new compounds not observed by pyrolysis in the absence of TMAH were produced. Their mass spectra, with characteristic fragments at $m / z 88$ and 101, suggest that they consist of permethylated monosaccharides, although no definitive structures have been proposed for them.

In agreement with the mechanism proposed by Challinor [7-9] and de Leeuw and Bass [10], there are few reasons to assume that a reaction between the macromolecule and TMAH occurs at the pyrolysis temperature which would release the structural units upon pyrolysis. In fact, the reported findings are in general very different to the results obtained by direct methylation followed by Py [5].

\section{CONCLUSIONS}

A set of fulvic acids (FA) from different origins were pyrolysed both in the absence and in the presence of TMAH. The pyrolysis of FA in presence of TMAH yielded (among other typical Py products FA, such as derivatives from polysaccharides and lignin) some new Py products that have been detected before only by drastic and time-consuming chemical degradation procedures, and that could probably arise from the building blocks of the FA macromolecular structures. This procedure aslo releases moieties that have not previously been observed upon pyrolysis because of being too polar or too reactive. The results obtained suggest a thermally assisted chemolysis rather than a methylation of the pyrolysis products.

Although, because of the complex chemical nature of FAs, we have not assessed the extent of the potential mechanisms involved, we believe that the chemical characterization of FAs at the molecular level can be accomplished by using this new approach, which simplifies sample preparation and also reduces the time and the amount of sample required for analysis. Pyrolysis in the presence of TMAH seems to be a suitable technique for the structural elucidation of humic macromolecules based on benzenecarboxylic acids.

\section{REFERENCES}

I F.H. Frimmel and R.F. Christman (Eds.), Humic Substances and their Role in the Environment, Wiley, Chichester, 1988, p. 271.

2 P. MacCarthy, C.E. Clapp, R.L. Matcolm and P.R. Bloom (Eds.), Humic Substances in Soil and Crop Sciences: Selected Readings, Am. Soc. Agrom., Madison, 1990, p. 281.

3 K. Anderson and R. Winans, Anal Chem., 63 (1991) 2901.

4 F. Martin and F.J. Gonzàlez-Vila, Z. Pflanzenernachr. Bodenkd., 146 (1983) 653. 
5 F. Martin and F.J. González-Vila, in B. Allard, H. Borem and A. Grinvall (Eds.), Lectures Notes in Earth Sciences, 33, Humic Substances in the Aquatic and Terrestrial Environment, Springer, Berlin, 1991, p. 105.

6 G. Almendros, F. Martin, F.J. González-Vila and J.C. del Rio. J. Anal. Appl. Pyrolysis, 25 (1993) 137.

7 J.M. Challinor, J. Anal. Appl. Pyrolysis, 16 (1989) 323.

8 J.M. Challinor, J. Anal. Appl. Pyrolysis, 18 (1991) 233.

9 J.M. Chaltinor, J. Anal. Appl. Pyrolysis, 20 (1991) is.

10 J.W. de Leeuw and M. Baas, J. Anal. Appl. Pyrolysis, 26 (1993) 175.

11 F. Martin and F.J. González-Vila, Chem. Geol., 67 (1988) 353.

12 F.J. González-Vila, U. Kaatze, H. Lentz, F. Martin and R. Pottel, in B. Allard, H. Borem and A. Grinvall (Eds.), Lectures Notes in Earth Sciences, 33, Humic Substances in the Aquatic and Terrestrial Environment, Springer, Berlin, 1991, p. 75-83.

13 M. Schnitzer and S.I.M. Skinner, Soil Sci., 105 (1968) 392.

14 F. Martin and F.J. González-Vila, A. Pflanzenernachr. Bodenkd., 146 ( 1983 ) 409.

15 M. Schnitzer and S.U. Kahn (Eds.), Soil Organic Matter, Elsevier, Amsterdam, 1978, Chapter 1, p. 26.

16 F.J. Stevenson, Humus Chemistry, Wiley, New York, 1978, Chapter 5, p. 120. 